\title{
Fiber absorption measurement errors resulting from re-emission of radiation
}

\author{
Betty Meng Zhang ${ }^{1,2}$, Junqing Zhao ${ }^{1}$, Yutong Feng ${ }^{1}$, Sheng Zhu ${ }^{1}$, Jonathan H. V. Price ${ }^{1}$, and Johan Nilsson ${ }^{1, *}$ \\ 1) Optoelectronics Research Centre, University of Southampton, Southampton SO17 1BJ, UK \\ 2) Also with School of Electrical and Electronic Engineering, Nanyang Technological University, Singapore 639798, Singapore \\ *jn@orc.soton.ac.uk
}

\begin{abstract}
: absorption and emission spectra.

OCIS codes: (300.0300) Spectroscopy; (060.2270) Fiber characterization; (300.1030) Absorption
\end{abstract}

We show that errors in the absorption measured in rare-earth-doped fibers can exceed $50 \%$ and severely distort the spectral shape. This is a result of re-emission in fibers with overlapping

\section{Introduction}

The spectral shape and the size of the absorption is a key characteristic of any laser medium. While it is rather straightforward to measure, several factors can impair the accuracy. For example, if the absorption is measured with excessive power, the fraction of excited laser ions can become large enough to modify the size and spectral shape of the absorption. There may also be nonlinear wavelength conversion, e.g., if a supercontinuum source with high peak power is used for the measurements. These measurement errors are particularly likely to occur in a fiber, because of the tight confinement and because a long interaction length promotes nonlinear wavelength conversion.

Furthermore, in multimode structures such as double-clad fibers, differences in the absorption between different modes can also distort the absorption spectrum (e.g., $[1,2])$. This is not a measurement error but rather a true effect that reduces the absorption in longer fibers due to different overlap between different pump modes and the doped region and inadequate mode mixing. These effects are especially strong for high absorption values, and therefore they alter the absorption peak most strongly.

In this paper, we investigate another effect that leads to errors in absorption measurements, namely re-emission of absorbed light at wavelengths that overlap with the absorption line under study. This can be important when a large fraction of the emission can reach the detector such as with high-NA fibers, when the laser dopant can absorb or emit over a large range of wavelengths, and when the spectral filtering of the probe light occurs at either the source or the detector end of the measurement setup, but not at both ends. For low absorption values, the probe photons absorbed by the dopant can to a significant fraction be compensated for by re-emitted photons. For high absorption values, the few photons that reach the detector can be dominated by re-emitted photons rather than by probe photons. In the high-absorption regime, re-emission affects the peak in particular, and is thus another effect that also leads to the often-seen reduction of the measured peak absorption at high absorption levels. We use singlemode fibers to eliminate the influence of differential mode absorption. For example, simulations show that in a single-mode $\mathrm{Yb}$-doped fiber with $\mathrm{NA}=0.24$ and $\mathrm{V}=2$, the peak absorption could actually be $82 \mathrm{~dB}$ when the measured value is $33 \mathrm{~dB}$.

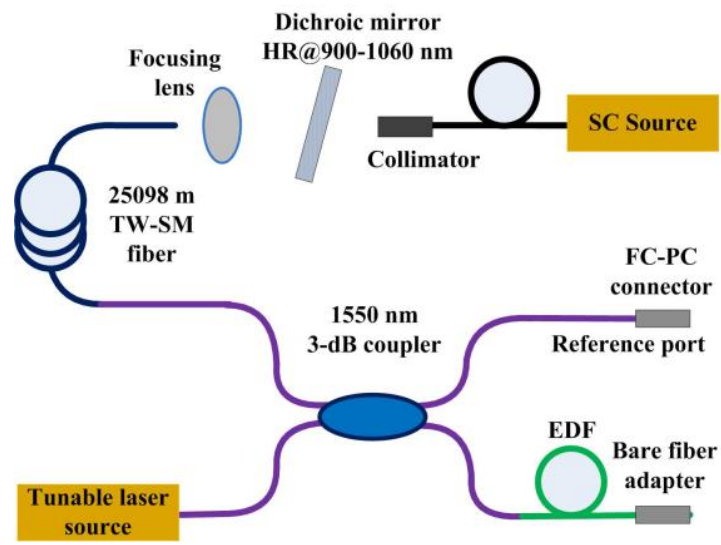

Fig. 1. Absorption measurement setup. EDF: Er-doped fiber.

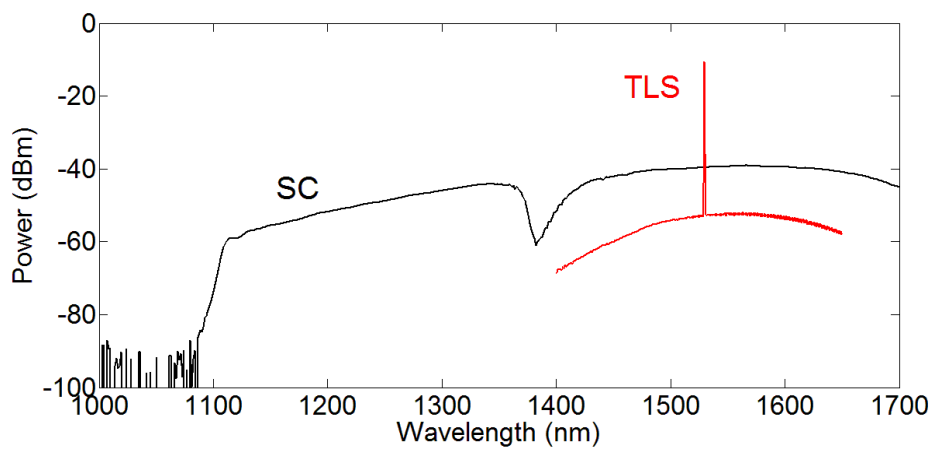

Fig. 2. Spectrum of supercontinuum-source and TLS as launched into the EDF.

Figure 1 illustrates the configuration we primarily consider. This is a conventional setup for fiber absorption measurements. We have a choice between a white-light source (WLS, in our case a Fianium supercontinuum source) 
or a tunable laser source (TLS, Agilent 8164A), as well as between an Ando AQ6317B optical spectrum analyzer (OSA) and a broadband power meter (HP 8153A with HP81525 sensor). In most cases we use a WLS and an OSA for these measurements. This approach was used for all measurements of absorption spectra, with the OSA set to 1 $\mathrm{nm}$ resolution. The detected signal was in every case unaffected by receiver noise, so that clean traces were obtained. A dichroic mirror and a $25-\mathrm{km}$ long standard single-mode fiber removed short wavelengths from the supercontinuum spectrum. Figure 2 shows the spectra of the TLS and supercontinuum (SC) source, as launched into the fiber under test (an Er-doped fiber, EDF, in Fig. 1).

\section{Results}

We measured the absorption of varying lengths of a single-mode EDF (Fibercore I-12(980/125) HC). It had a coreNA of 0.25 and a mode-field diameter of between 5 and $5.5 \mu \mathrm{m}$. The peak absorption at $\sim 1530 \mathrm{~nm}$ was $\sim 21 \mathrm{~dB} / \mathrm{m}$. Figure 3 shows how the measured absorption, as determined from the light launched into and exiting the EDF, depends on the fiber length for three different measurement methods. The best method is to use the TLS combined with the OSA, to achieve spectral selection at both source and detector. In this case, the measured absorption is proportional to fiber length over the entire measurement range up to a peak absorption of $58 \mathrm{~dB}$. By contrast, with a WLS instead of the TLS, the measured absorption only reaches $\sim 33 \mathrm{~dB}$, which is only $57 \%$ of the actual value with the $2.8 \mathrm{~m}$ fiber length. With the TLS combined with the broadband power meter, the measured absorption only reaches $\sim 21 \mathrm{~dB}$.

Figure 4 shows absorption spectra for different fiber lengths as determined through cutback, with an $11-\mathrm{cm}$ length of EDF providing the reference spectrum in the cutback calculations. The absorption peak is severely distorted. We attribute the distortion and the reduction in measured absorption to photons that are wavelengthconverted in the fiber. In the case of the WLS, photons launched into the fiber at low-absorbing wavelengths can propagate through a large fraction of the fiber in significant numbers. They are then absorbed, closer to the output end, and remitted at the absorption peak with a probability that is relatively high, since the fluorescence spectrum peaks near the absorption peak. The re-emitted photons couple into the forward-propagating mode with probability approximately equal to $\mathrm{NA}^{2} / 4$. At this wavelength the absorption is high, but since they are now closer to the detector they still reach it in significant numbers. At the absorption peak, such re-emitted photons can then dominate over the few probe-photons that travel through the fiber without absorption. A corresponding process occurs in the case of a TLS combined with a broadband detector. Insofar as the excitation of Er is small, these effects are powerindependent.

The near-complete elimination of the absorption peak with minimal distortion away from the peak is striking and qualitatively different from the spectral distortions resulting from the use of excessive power (when the Er-excitation is non-negligible) and perhaps also from the case of differential modal absorption in double-clad fibers. This may be due to the fact that most photons are re-emitted at the peak.

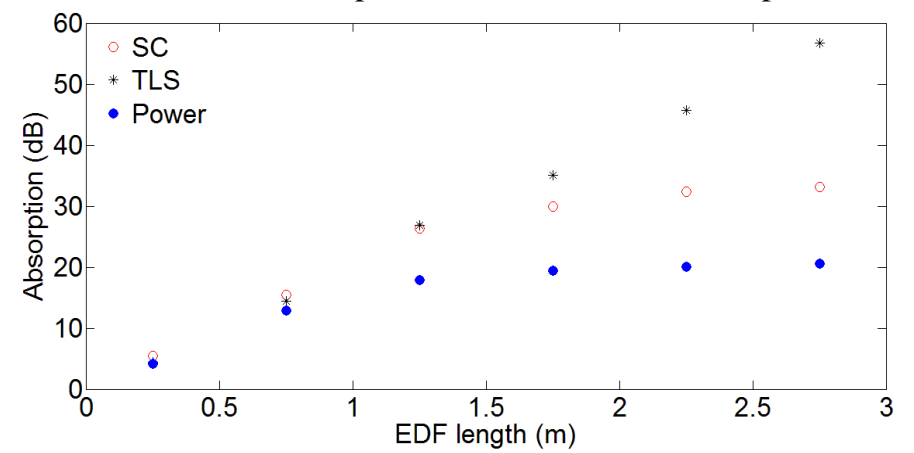

Fig. 3. Absorption at $1530 \mathrm{~nm} v s$. fiber length measured with three different methods. "Power": absorption measured with a TLS and a broadband detector.

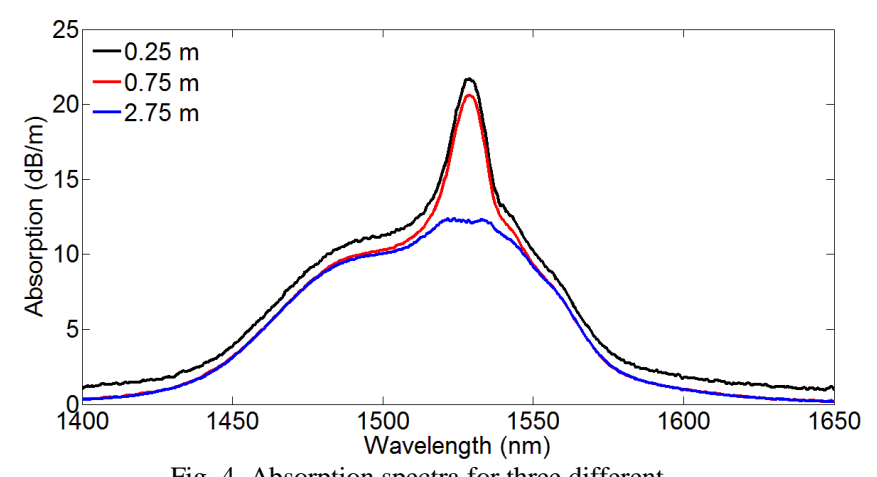

Fig. 4. Absorption spectra for three different EDF cutback lengths measured with a WLS.

With WLS excitation, also absorption into higher-lying levels matter. Erbium has a number of shorter-wavelength absorption bands, and photons absorbed by them would also be re-emitted into the 1.5 - $\mu \mathrm{m}$ band with high quantum efficiency. This is a particular concern if the spectral power density is higher at shorter wavelengths. In our case, however, it is clear from Fig. 2 that there is no significant power at short wavelengths, so the only significant absorption will be into the $1.5-\mu \mathrm{m}$ band. The total power launched into the Er-doped fiber is around $10 \mu \mathrm{W}$, which is low enough for the fraction of excited Er-ions to be negligible. The spectrum of the TLS is shown, too. There is significant background power extending to wavelengths where the Er-absorption is small. This contributes to the transmitted power as measured by the broadband detector, which explains the poor performance in Fig. 3 in that 
case. The total power launched into the Er-doped fiber from the TLS is around $100 \mu \mathrm{W}$, which is just about low enough for the fraction of excited Er-ions to be negligible.

Figure 5 shows the error in the peak absorption as determined by white-light absorption characteristics. These are the results of simulations with RP Fiber Power in the case of white light being launched into the fiber over the absorption-band under study. For an EDF with an NA of 0.24 and a V-number of 2, the error is $38 \%$ at an actual absorption of $58 \mathrm{~dB}$. This agrees well with the measurements in Fig. 3. Simulation results with single-mode $\mathrm{Yb}-$ doped fibers are shown, too. They show larger errors than the EDFs. We attribute this to a wider absorption spectrum of $\mathrm{Yb}$, a sharper peak, and / or a higher radiative quantum efficiency as implicitly determined by the fluorescence lifetime used in the simulations.

The NA also makes a big difference (Fig. 5). While an NA of 0.46 is highly unusual for a single-mode fiber, an NA of 0.24 is quite common and still leads to large errors already at low absorptions. On the other hand, largemode-area fibers can have an NA of, e.g., 0.06, which is lower than what is considered in Fig. 5. Re-emission effects will often be negligible in practice for such low NAs.

Figure 5 also shows an error for short fibers, in which the absorption is low. Also in this case the reason is that absorbed photons are re-emitted and then recaptured by the guided mode and propagated to the detector. In contrast to the high-absorption case, this error will increase at longer wavelengths, where the emission-to-absorption crosssection ratio is large. Finally, Fig. 6 shows a simulated white-light absorption spectrum, together with a reference one calculated from the absorption cross-section spectrum. As for the experimental curves in Fig. 3, the absorption peak virtually disappears in case of simulated white-light measurement with high absorption, while errors away from the peak are small.

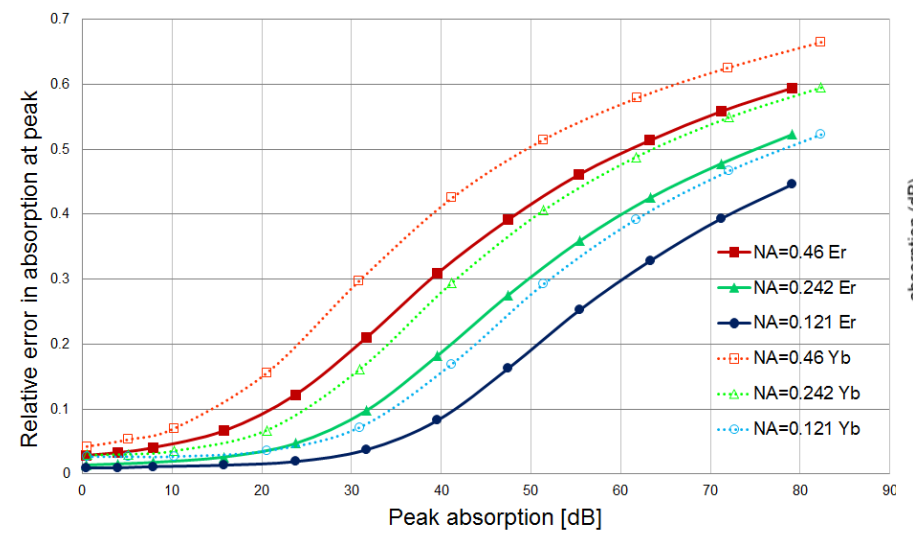

Fig. 5. Relative error in peak absorption $v s$. actual absorption for single-mode $(\mathrm{V}=2) \mathrm{Er}$ and $\mathrm{Yb}$-doped fibers of different NA. Results of numerical simualtions.

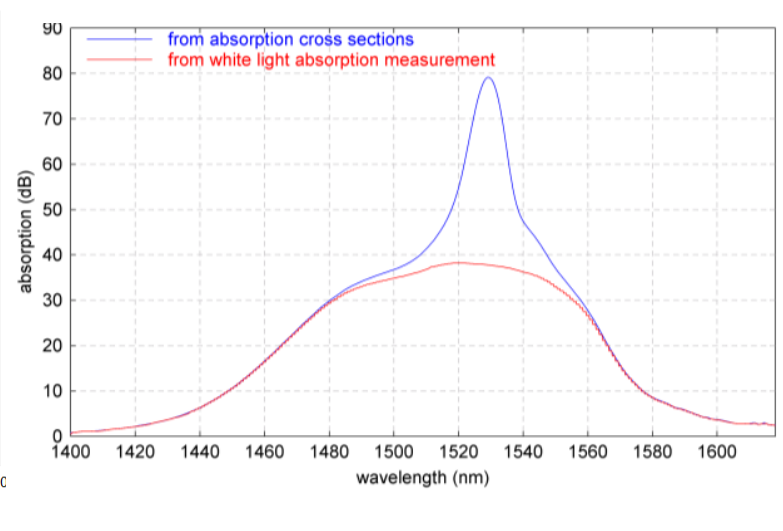

Fig. 6. Intrinsic absorption spectrum (given by the absorption cross-sections and the overlap) and distorted spectrum obtained from simulations of white-light absorption.

\section{Conclusions}

Simulations as well as measurements show that re-emission effects can lead to unacceptable errors in the characterization of absorption of rare-earth doped fibers with spectrally overlapping emission and absorption when using broadband probe / excitation sources. The effect increase with the NA and with higher absorption values, but an error remains also at low absorption. We expect similar errors will occur also in non-fiber geometries as well as in double-clad fibers, if re-emitted photons are collected with a similarly high NA. Preliminary measurements on double-clad fibers show similar effects.

Re-emission effects can be mitigated by the use of a narrow-line probe together with narrow-line detection (the narrower the better), as well as by time-gating to preferentially select ballistic photons over re-emitted photons.

Acknowledgement: This work was supported by funding from EPSRC (UK) Centre for Innovative Manufacturing in Photonics EP/H02607X/1. The data for this paper can be found at http://dx.doi.org/10.5258/SOTON/380319

\section{References}

[1] A. Liu \& K. Ueda, "The absorption characteristics of circular, offset, and rectangular double-clad fibers," Opt. Commun. 132, 511-518 (1996)

[2] M. N. Zervas and C. A. Codemard, "High Power Fiber Lasers: A Review," IEEE J. Sel. Top. Quantum Electron. 20, 219-241 (2014) 